\title{
О числе представлений натуральных чисел суммами девяти квадратов
}

\author{
Г. А. ЛОмадЗЕ (Тбилиси)
}

1. Впервые Морделл [6], применив модулярные функции, развил метод нахождения точных (неасимптотических) формул для числа представлений натуральных чисел суммами нечетного числа квадратов целых чисел. Проделав соответствующие вычисления, впервые он получил формулы для числа представлений натуральных чисел суммами 11, 13 и 15 квадратов целых чисел. Дополнительные члены этих формул оказались некоторыми суммами, распространенными на все представления данного числа суммой 3, 5 и 7 квадратов целых чисел соответственно. Морделл также замечает, что если применить известное тождество Якоби $\vartheta_{1}^{\prime}=\vartheta_{2} \vartheta_{3} \vartheta_{0}$, то дополнительный член в формуле для числа представлений суммой 9 квадратов можно выразить при помощи числа представлений суммой 7 квадратов. Согласно этому замечанию, в [3] показано, что число представлений натурального числа $n$ суммой 9 квадратов равно

$$
r_{9}=\varrho_{9}(n)+\frac{2}{17} \sum_{\substack{x_{1}^{2}+\ldots+x_{7}^{2}=4 n \\ 2\left|x_{2}, 2\right| x_{3}, 2 \mid x_{4} \\ 2 \nmid x_{1}, 2 \nmid x_{5}, 2 \nmid x_{6}, 2 \nmid x_{7}}}(-1)^{\left(x_{1}+x_{2}+x_{3}+x_{4}-1\right) / 2} x_{1} .
$$

Эта формула малоинтересна. Ввиду этого в [4], вместо упомянутой выше формулы Якоби, применено тождество Смита

$$
\frac{\vartheta_{0}^{\prime \prime}}{\vartheta_{0}}-\frac{\vartheta_{3}^{\prime \prime}}{\vartheta_{3}}=\vartheta_{2}^{4}
$$

и показано, что

$$
r_{9}(n)=\varrho_{9}(n)+\frac{8}{17} \sum_{x_{1}^{2}+\ldots+x_{5}^{2}=n}(-1)^{x_{1}+x_{2}+x_{3}+x_{4}}\left(x_{5}^{2}-x_{4}^{2}\right) .
$$

В настоящей же статье, при помощи результатов работы [5], 
будет доказано, что

$$
r_{9}(n)=\varrho_{9}(n)+\frac{32}{17} \sum_{\substack{x_{1}^{2}+x_{2}^{2}+x_{3}^{2}=3 n \\ 3 \nmid x_{1}, 3 \nmid x_{2}, 3 \nmid x_{3} \\ x_{1}>0, x_{2}>0, x_{3}>0}}\left(\frac{x_{1} x_{2} x_{3}}{3}\right) x_{1} x_{2} x_{3} .
$$

Если положить $n=2^{k} u(k \in \mathbb{Z}, k \geq 0), u=\prod_{p \mid u} p^{l}(l \in \mathbb{Z}, l \geq 1)$, $\nu=p^{-l} n, n=r^{2} \omega(\omega-$ бесквадратное), то во всех приведенных выше трех формулах

$$
\varrho_{9}(n)=\frac{24576}{17 \pi^{4}} n^{7 / 2} \chi_{2}(n) T_{4}(n) \mathcal{L}(4, \omega),
$$

где

$$
\begin{aligned}
& \chi_{2}(n)= \begin{cases}\frac{135}{127}+\frac{119}{127 \cdot 128} 2^{-7 k / 2} & \text { при } 2 \mid k, u \equiv 1(\bmod 8), \\
\frac{135}{127}-\frac{135}{127 \cdot 128} 2^{-7 k / 2} & \text { при } 2 \mid k, \omega \equiv 5(\bmod 8), \\
\frac{135}{127}-\frac{255}{127 \cdot 16} 2^{-7 k / 2} & \text { при } 2 \mid k, \omega \equiv 3(\bmod 4), \\
\frac{135}{127}-\frac{255}{127 \cdot 16} 2^{-7(k-1) / 2} & \text { при } 2 \nmid k ;\end{cases} \\
& T_{4}(n)=\prod_{p \mid r, p>2}\left(1-\left(\frac{\omega}{p}\right) p^{-4}\right) \prod_{p \mid u}\left(1-p^{-7}\right)^{-1} \prod_{p \mid u, 2 \nmid l}\left(1-p^{-7(l+1) / 2}\right) \\
& \times \prod_{p|u, 2| l}\left(1+\frac{\left(\frac{\nu}{p}\right)-p^{-3}}{1-\left(\frac{\nu}{p}\right) p^{-4}} p^{-7 k / 2-4}\right) \\
& \mathcal{L}(4,1)=\frac{\pi^{4}}{96}, \quad \mathcal{L}(4,2)=\frac{11 \pi^{4}}{768 \sqrt{2}}, \\
& \mathcal{L}(4, \omega)=-2 \pi^{4} \omega^{-1 / 2} \sum_{0 \leq h \leq \omega / 2}\left(\frac{h}{\omega}\right)\left(\frac{h^{2}}{4 \omega^{2}}-\frac{h^{3}}{3 \omega^{3}}\right) \\
& \text { при } \omega \equiv 1(\bmod 4), \omega>1 \text {, } \\
& \mathcal{L}(4, \omega)=2 \pi^{4} \omega^{-1 / 2}\left\{\sum_{0<h \leq \omega / 4}\left(\frac{h}{\omega}\right)\left(\frac{h}{16 \omega}-\frac{h^{3}}{3 \omega^{3}}\right)\right. \\
& \left.-\sum_{\omega / 4<h \leq \omega / 2}\left(\frac{h}{\omega}\right)\left(\frac{1}{96}-\frac{3 h}{16 \omega}+\frac{h^{2}}{2 \omega^{2}}-\frac{h^{3}}{3 \omega^{3}}\right)\right\} \\
& \text { при } \omega \equiv 3(\bmod 4) \text {, }
\end{aligned}
$$




$$
\begin{aligned}
\mathcal{L}(4, \omega)= & 2 \pi^{4} \omega^{-1 / 2}\left\{\sum_{0<h \leq \omega / 16}\left(\frac{h}{\omega / 2}\right)\left(\frac{11}{768}-\frac{h^{2}}{\omega^{2}}\right)\right. \\
& +\sum_{\omega / 16<h \leq 3 \omega / 16}\left(\frac{h}{\omega / 2}\right)\left(\frac{5}{384}+\frac{h}{16 \omega}-\frac{2 h^{2}}{\omega^{2}}+\frac{16 h^{3}}{3 \omega^{3}}\right) \\
& \left.+\sum_{3 \omega / 16<h \leq \omega / 4}\left(\frac{h}{\omega / 2}\right)\left(\frac{37}{768}-\frac{h}{2 \omega}+\frac{h^{2}}{\omega^{2}}\right)\right\}
\end{aligned}
$$

при $\omega \equiv 2(\bmod 8), \omega>2$,

$$
\begin{aligned}
& \mathcal{L}(4, \omega)= 2 \pi^{4} \omega^{-1 / 2}\left\{\sum_{0<h \leq \omega / 16}\left(\frac{h}{\omega / 2}\right)\left(\frac{3 h}{16 \omega}-\frac{16 h^{3}}{3 \omega^{3}}\right)\right. \\
&-\sum_{\omega / 16<h \leq 3 \omega / 16}\left(\frac{h}{\omega / 2}\right)\left(\frac{1}{768}-\frac{h}{4 \omega}+\frac{h^{3}}{\omega^{3}}\right) \\
&\left.-\sum_{3 \omega / 16<h \leq \omega / 4}\left(\frac{h}{\omega / 2}\right)\left(\frac{7}{192}-\frac{13 h}{16 \omega}+\frac{4 h^{2}}{\omega^{2}}-\frac{16 h^{3}}{3 \omega^{3}}\right)\right\} \\
& \text { при } \omega \equiv 6(\bmod 8) .
\end{aligned}
$$

2. В настоящей статье будут применяться следующие обозначения: $N, a, n, q, r$ - натуральные числа; $\omega$ - бесквадратное число; $s \geq 5, u$ - нечетные натуральные числа; $p$ - простое число; $c, g, h, j$, $k, l, m, x, \alpha, \beta, \gamma, \delta$ - целые числа; $A, B, \lambda$ - комплексные числа; $z, \tau$ - комплексные переменные $(\operatorname{Im} \tau>0)$; для $z \neq 0$ положим: $z^{s / 2}=$ $\left(z^{1 / 2}\right)^{s},-\pi / 2<\arg z^{1 / 2} \leq \pi / 2$. Далее, $\left(\frac{h}{u}\right)$ обозначает обобщенный симбол Якоби; $e(z)=\exp (2 \pi i z) ; \eta(\gamma)=1$ при $\gamma \geq 0$ и $\eta(\gamma)=-1$ при $\gamma<0 ; S(h, q)$ - сумма Гаусса; $r\left(n ; f_{s}\right)$ - число представлений числа $n$ примитивной положительной квадратичной формой $f_{s}=$ $a_{1} x_{1}^{2}+\ldots+a_{s} x_{s}^{2}$ определителя $\Delta, a$ - общее наименьшее кратное коэффициентов $a_{k}(k=1,2, \ldots, s) ; \varrho\left(n ; f_{s}\right)$ - сумма сингулярного ряда, соответствующего квадратичной форме $f_{s}$.

Далее, пусть

$$
\begin{aligned}
& \vartheta_{g h}(z \mid \tau ; c, N) \\
& \quad=\sum_{m \equiv c(\bmod N)}(-1)^{h(m-c) / N} e\left((2 m+g)^{2} \tau /(8 N)\right) e((2 m+g) z / 2) .
\end{aligned}
$$

Положим

$$
\begin{aligned}
& \vartheta_{g h}(\tau ; c, N)=\vartheta_{g h}(0 \mid \tau ; c, N), \\
& \vartheta_{g h}^{\prime}(\tau ; c, N)=\left.\frac{\partial}{\partial z} \vartheta_{g h}(z \mid \tau ; c, N)\right|_{z=0} .
\end{aligned}
$$


Известно ([2], с. 318, форм. (1.2), (1.4)), что

$$
\begin{aligned}
\vartheta_{g+2 j, h}(z \mid \tau ; c, N) & =\vartheta_{g h}(z \mid \tau ; c+j, N), \\
\vartheta_{g h}(z \mid \tau ; c+N j, N) & =(-1)^{h j} \vartheta_{g h}(z \mid \tau ; c, N) .
\end{aligned}
$$

Следовательно, согласно (2.2),

$$
\begin{aligned}
\vartheta_{g+2 j, h}^{\prime}(\tau ; c, N) & =\vartheta_{g h}^{\prime}(\tau ; c+j, N), \\
\vartheta_{g h}^{\prime}(\tau ; c+N j, N) & =(-1)^{h j} \vartheta_{g h}^{\prime}(\tau ; c, N) .
\end{aligned}
$$

Из (2.1), согласно (2.2), в частности получим

$$
\begin{aligned}
& \vartheta_{g h}(\tau ; 0, N)=\sum_{m=-\infty}^{\infty}(-1)^{h m} e\left((2 N m+g)^{2} \tau /(8 N)\right), \\
& \vartheta_{g h}^{\prime}(\tau ; 0, N)=\pi i \sum_{m=-\infty}^{\infty}(-1)^{h m}(2 N m+g) e\left((2 N m+g)^{2} \tau /(8 N)\right) .
\end{aligned}
$$

Из (2.6) следует, что

$$
\vartheta_{-g h}^{\prime}(\tau ; 0, N)=-\vartheta_{g h}^{\prime}(\tau ; 0, N) .
$$

Из (2.5) следует

$$
\prod_{k=1}^{s} \vartheta_{00}\left(\tau ; 0,2 a_{k}\right)=1+\sum_{n=1}^{\infty} r\left(n ; f_{s}\right) e(n \tau) .
$$

Далее, положим

$$
\theta\left(\tau ; f_{s}\right)=1+\sum_{n=1}^{\infty} \varrho\left(n ; f_{s}\right) e(n \tau)
$$

где

(2.10) $\varrho\left(n ; f_{s}\right)=\frac{\pi^{s / 2}}{\Gamma(s / 2) \Delta^{1 / 2}} n^{s / 2-1} \sum_{q=1}^{\infty} q^{-s} \sum_{\substack{h \bmod \\(h, q)=1}} e\left(-\frac{n h}{q}\right) \prod_{k=1}^{s} S\left(a_{k} h, q\right)$.

Наконец, пусть

$\Gamma\left\{\frac{\alpha \tau+\beta}{\gamma \tau+\delta} \mid \alpha \delta-\beta \gamma=1\right\}, \quad \Gamma_{0}(4 N)=\left\{\frac{\alpha \tau+\beta}{\gamma \tau+\delta} \in \Gamma \mid \gamma \equiv 0(\bmod 4 N)\right\}$.

ОпределениЕ. Функцию $F$, определенную на $\mathcal{H}=\{\tau \in \mathbb{C} \mid$ $\operatorname{Im} \tau>0\}$, будем называть челой модулярной формой веса $s / 2$ и системы мультипликаторов $v(M)$ относительно подгруппы $\Gamma_{0}(4 N)$ $\left(M-\right.$ матрица произвольной подстановки из $\left.\Gamma_{0}(4 N)\right)$, если

(1) $F$ регулярна на $\mathcal{H}$, 
(2) для всех матриц $M=\left(\begin{array}{ll}\alpha & \beta \\ \gamma & \delta\end{array}\right)$ подстановок из $\Gamma_{0}(4 N)$ и всех $\tau \in \mathcal{H}$

$$
F\left(\frac{\alpha \tau+\beta}{\gamma \tau+\delta}\right)=v(M)(\gamma \tau+\delta)^{s / 2} F(\tau),
$$

(3) в окрестности точки $\tau=i \infty$,

$$
F(\tau)=\sum_{m=0}^{\infty} A_{m} e(m \tau),
$$

(4) для любой подстановки из $\Gamma$, в окрестности каждой рациональной точки $\tau=-\delta / \gamma(\gamma \neq 0,(\gamma, \delta)=1)$,

$$
(\gamma \tau+\delta)^{s / 2} F(\tau)=\sum_{m=0}^{\infty} B_{m} e\left(\frac{m}{4 N} \frac{\alpha \tau+\beta}{\gamma \tau+\delta}\right) .
$$

Пусть $\left\{\Gamma_{0}(4 N), s / 2, v(M)\right\}$ обозначает множество всех таких целых модулярных форм $F$.

Лемма 1 ([1], с. 811, 953). Функция $F$ из множества $\left\{\Gamma_{0}(4 N)\right.$, $s / 2, v(M)\}$ тождественно равна нулю, если в ее разложении (2.11),

$$
A_{m}=0 \quad \text { для всех } m \leq \frac{s}{24} 4 N \prod_{p \mid 4 N}\left(1+\frac{1}{p}\right) .
$$

Лемма 2 ([5], теорема). Пусть $N \geq a$. Функция

$$
\psi\left(\tau ; f_{9}\right)=\prod_{k=1}^{9} \vartheta_{00}\left(\tau ; 0,2 a_{k}\right)-\theta\left(\tau ; f_{9}\right)-\lambda \prod_{k=1}^{3} \vartheta_{g_{k} h_{k}}^{\prime}\left(\tau ; 0,2 N_{k}\right),
$$

где $\lambda$ - произвольная постоянная, принадлежит множеству $\left\{\Gamma_{0}(4 N)\right.$, $9 / 2, v(M)\}\left(M=\left(\begin{array}{cc}\alpha & \beta \\ \gamma & \delta\end{array}\right)-\right.$ матрица подстановки из $\Gamma_{0}(4 N), v(M)=$ $\left.i^{\eta(\gamma)(\operatorname{sgn} \delta-1) / 2} \cdot i^{(|\delta|-1)^{2} / 4}(\beta \Delta \operatorname{sgn} \delta /|\delta|)\right)$, если выполняются следующие условия:

(1) $2\left|g_{k}, N_{k}\right| N(k=1,2,3), a \mid N$,

(2) $4\left|N \sum_{k=1}^{3} \frac{h_{k}^{2}}{N_{k}}, 4\right| \sum_{k=1}^{3} \frac{g_{k}^{2}}{4 N_{k}}$,

(3) для всех $\alpha$ и $\delta$, для которых $\alpha \delta \equiv 1(\bmod 4 N)$,

$$
\operatorname{sgn} \delta\left(\frac{N_{1} N_{2} N_{3}}{|\delta|}\right) \prod_{k=1}^{3} \vartheta_{\alpha g_{k}, h_{k}}^{\prime}\left(\tau ; 0,2 N_{k}\right)=\left(\frac{-\Delta}{|\delta|}\right) \prod_{k=1}^{3} \vartheta_{g_{k} h_{k}}^{\prime}\left(\tau ; 0,2 N_{k}\right) \text {. }
$$

3. В этом параграфе будет выведена формула (1.1) для числа представлений натурального числа $n$ квадратичной формой $f_{9}=$ 
$x_{1}^{2}+\ldots+x_{9}^{2}$ определителя $\Delta=a=1$. Как показано в [3], с. 297-299, в этом случае формула (2.10) будет иметь вид (1.2).

Лемма 3. Функиия

$$
\psi(\tau)=\vartheta_{00}^{9}(\tau ; 0,2)-\theta_{9}(\tau)-\frac{i}{34 \pi^{3}} \vartheta_{40}^{\prime 3}(\tau ; 0,6)
$$

принадлежит множеству $\left\{\Gamma_{0}(12), 9 / 2, v(M)\right\}$, әде

$$
v(M)=i^{\eta(\gamma)(\operatorname{sgn} \delta-1) / 2} \cdot i^{(|\delta|-1)^{2} / 4}\left(\frac{\beta \operatorname{sgn} \delta}{|\delta|}\right),
$$

$M=\left(\begin{array}{cc}\alpha & \beta \\ \gamma & \delta\end{array}\right)-$ матрица подстановки $\in \Gamma_{0}(12)$.

Доказательство. 1 . В лемме 2 положим: $\psi\left(\tau ; f_{9}\right)=\psi(\tau) ; f_{9}=$ $x_{1}^{2}+\ldots+x_{9}^{2}$, т.е. $a_{1}=\ldots=a_{9}=a=\Delta=1 ; \theta\left(\tau ; f_{9}\right)=\theta_{9}(\tau)=$ $1+\sum_{n=1}^{\infty} \varrho_{9}(n) e(n \tau) ; \lambda=i /\left(34 \pi^{3}\right) ; g_{1}=g_{2}=g_{3}=4, h_{1}=h_{2}=h_{3}=0$, $N_{1}=N_{2}=N_{3}=N=3$. Нетрудно проверить, что функция $\psi(\tau)$ удовлетворяет условиям (1) и (2) леммы 2.

2. Из $\alpha \delta \equiv 1(\bmod 12)$ следует, что

$$
\alpha \equiv \delta \equiv \pm 1, \pm 5(\bmod 12) ;
$$

следовательно, $\alpha \equiv \pm 1(\bmod 3)$ и, согласно $(2.3),(2.4)$ и $(2.7)$, получим

$$
\vartheta_{4 \alpha, 0}^{\prime 3}(\tau ; 0,6)=\vartheta_{ \pm 4+4(\alpha \mp 1), 0}^{\prime 3}(\tau ; 0,6)
$$

$$
\begin{aligned}
& =\vartheta_{ \pm 4,0}^{\prime 3}(\tau ; 2(\alpha \mp 1), 6)=\vartheta_{ \pm 4,0}^{\prime 3}(\tau ; 0,6) \\
& = \begin{cases}\vartheta_{40}^{\prime 3}(\tau ; 0,6) & \text { при } \alpha \equiv 1(\bmod 3), \text { т.е. при } \alpha \equiv 1,-5(\bmod 12), \\
-\vartheta_{40}^{\prime 3}(\tau ; 0,6) & \text { при } \alpha \equiv-1(\bmod 3), \text { т.е. при } \alpha \equiv-1,5(\bmod 12) .\end{cases}
\end{aligned}
$$

Далее, при $\delta>0$ имеем

$$
\begin{gathered}
\operatorname{sgn} \delta\left(\frac{3 \cdot 3 \cdot 3}{|\delta|}\right)=\left(\frac{3}{\delta}\right)= \begin{cases}1, & \text { если } \delta \equiv \pm 1(\bmod 12), \\
-1, & \text { если } \delta \equiv \pm 5(\bmod 12),\end{cases} \\
\left(\frac{-1}{|\delta|}\right)=\left(\frac{-1}{\delta}\right)= \begin{cases}1, & \text { если } \delta \equiv 1,5(\bmod 12), \\
-1, & \text { если } \delta \equiv-1,-5(\bmod 12)\end{cases}
\end{gathered}
$$

при $\delta<0$ имеем

$$
\begin{gathered}
\operatorname{sgn} \delta\left(\frac{3 \cdot 3 \cdot 3}{|\delta|}\right)=-\left(\frac{3}{-\delta}\right)= \begin{cases}-1, & \text { если } \delta \equiv \pm 1(\bmod 12), \\
1, & \text { если } \delta \equiv \pm 5(\bmod 12),\end{cases} \\
\left(\frac{-1}{|\delta|}\right)=\left(\frac{-1}{-\delta}\right)= \begin{cases}1, & \text { если } \delta \equiv-1,-5(\bmod 12), \\
-1, & \text { если } \delta \equiv 1,5(\bmod 12)\end{cases}
\end{gathered}
$$


Следовательно,

$$
\operatorname{sgn} \delta\left(\frac{3}{|\delta|}\right)^{3}= \begin{cases}\left(\frac{-1}{|\delta|}\right) & \text { при } \delta \equiv 1,-5(\bmod 12), \\ -\left(\frac{-1}{|\delta|}\right) & \text { при } \delta \equiv-1,5(\bmod 12) .\end{cases}
$$

Из (3.2)-(3.4) следует, что

$$
\operatorname{sgn} \delta\left(\frac{3}{|\delta|}\right)^{3} \vartheta_{4 \alpha, 0}^{\prime 3}(\tau ; 0,6)=\left(\frac{-1}{|\delta|}\right) \vartheta_{40}^{\prime 3}(\tau ; 0,6),
$$

т.е. выполняется и условие (3) леммы 2 .

\section{Теорема. Имеет место тождество}

$$
\vartheta_{00}^{9}(\tau ; 0,2)=\theta_{9}(\tau)+\frac{i}{34 \pi^{3}} \vartheta_{40}^{\prime 3}(\tau ; 0,6) .
$$

Доказательство. Покажем, что в разложении функции $\psi(\tau)$ по степеням $Q=e(\tau)$ коэффициенты при $Q^{n}$ равны нулю для всех $n \leq 9$.

Из (2.5) следует, что

$$
\vartheta_{00}(\tau ; 0,2)=\sum_{m=-\infty}^{\infty} Q^{m^{2}}=1+2 Q+2 Q^{4}+2 Q^{9}+\ldots,
$$

откуда

$$
\begin{aligned}
\vartheta_{00}^{9}(\tau ; 0,2)= & +18 Q+144 Q^{2}+672 Q^{3}+2034 Q^{4}+4320 Q^{5} \\
& +7392 Q^{6}+12672 Q^{7}+22608 Q^{8}+34802 Q^{9}+\ldots
\end{aligned}
$$

Нетрудно проверить, что вычислив значения $\varrho_{9}(n)$ по формуле (1.2) для всех $n \leq 9$, получим

$$
\begin{aligned}
\theta_{9}(\tau)= & +\frac{274}{17} Q+\frac{2640}{17} Q^{2}+\frac{11040}{17} Q^{3}+\frac{34834}{17} Q^{4}+4320 Q^{5} \\
& +\frac{125280}{17} Q^{6}+\frac{216960}{17} Q^{7}+\frac{382800}{17} Q^{8}+\frac{592114}{17} Q^{9}+\ldots
\end{aligned}
$$

Из (2.6) следует

$$
\begin{aligned}
\vartheta_{40}^{\prime}(\tau ; 0,6) & =4 \pi i \sum_{m=-\infty}^{\infty}(3 m+1) Q^{(3 m+1)^{2} / 3} \\
& =4 \pi i\left(Q^{1 / 3}-2 Q^{4 / 3}+4 Q^{16 / 3}-5 Q^{25 / 3}+\ldots\right),
\end{aligned}
$$

откуда

$$
\begin{aligned}
& \vartheta_{40}^{\prime 3}(\tau ; 0,6)=64 \pi^{3} i^{3} Q\left(1-2 Q+4 Q^{5}-5 Q^{8}+\ldots\right)^{3} \\
& \quad=64 \pi^{3} i^{3} Q\left(1-6 Q+12 Q^{2}-8 Q^{3}+12 Q^{5}-48 Q^{6}+48 Q^{7}-15 Q^{8}+\ldots\right)
\end{aligned}
$$


следовательно,

$$
\begin{aligned}
& \frac{i}{34 \pi^{3}} \vartheta_{40}^{\prime 3}(\tau ; 0,6) \\
= & \frac{32}{17}\left(Q-6 Q^{2}+12 Q^{3}-8 Q^{4}+12 Q^{6}-48 Q^{7}+48 Q^{8}-15 Q^{9}+\ldots\right) .
\end{aligned}
$$

Приняв во внимание (3.1), (3.6), (3.7) и (3.10), нетрудно проверить, что коэффициенты при $Q^{n}$ в разложении функции $\psi(\tau)$ по степеням $Q$ равны нулю для всех $n \leq 9$. Следовательно, согласно лемме 1 , функция $\psi(\tau)$ тождественно равна нулю, т.е. тождество (3.5) доказано.

Доказательство формулы (1.1). Из (3.8) следует

$$
\begin{aligned}
& (3.11) \quad \frac{i}{64 \pi^{3}} \vartheta_{40}^{\prime 3}(\tau ; 0,6) \\
& =\sum_{m_{1}, m_{2}, m_{3}=-\infty}^{\infty}\left(3 m_{1}+1\right)\left(3 m_{2}+1\right)\left(3 m_{3}+1\right)
\end{aligned}
$$

$$
\times Q^{\left\{\left(3 m_{1}+1\right)^{2}+\left(3 m_{2}+1\right)^{2}+\left(3 m_{3}+1\right)^{2}\right\} / 3}
$$

$$
=\sum_{n=1}^{\infty}\left(\sum_{\left(3 m_{1}+1\right)^{2}+\left(3 m_{2}+1\right)^{2}+\left(3 m_{3}+1\right)^{2}=3 n}\left(3 m_{1}+1\right)\left(3 m_{2}+1\right)\left(3 m_{3}+1\right)\right) Q^{n} .
$$

Приравняв коэффициенты при $Q^{n}$ в обеих частях тождества (3.5) и приняв во внимание $(2.8),(2.9)$ и $(3.11)$, получим

$$
r_{9}(n)=\varrho_{9}(n)+\frac{32}{17} w(n),
$$

где $w(n)$ обозначает коэффициент при $Q^{n}$ в разложении функции $\frac{i}{64 \pi^{3}} \vartheta_{40}^{\prime 3}(\tau ; 0,6)$ по степеням $Q$, т.е.

$$
\begin{aligned}
w(n)= & \sum_{\begin{array}{c}
x_{1}^{2}+x_{2}^{2}+x_{3}^{2}=3 n \\
x_{1} \equiv x_{2} \equiv x_{3} \equiv 1(\bmod 3)
\end{array}} x_{1} x_{2} x_{3} \\
= & \sum_{\substack{x_{1}^{2}+x_{2}^{2}+x_{3}^{2}=3 n \\
3 \nmid x_{1}, 3 \nmid x_{2}, 3 \nmid x_{3} \\
x_{1}>0, x_{2}>0, x_{3}>0}}\left(\frac{x_{1} x_{2} x_{3}}{3}\right) x_{1} x_{2} x_{3} .
\end{aligned}
$$

Из (3.12) и (3.13) следует формула (1.1).

\section{Литература}

[1] E. Hecke, Mathematische Werke, zweite Auflage, Vandenhoeck u. Ruprecht, Göttingen, 1970. 
[2] H. D. Kloosterman, The behaviour of general theta-functions under the modular group and the characters of binary modular congruence groups. I, Ann. of Math. 47 (1946), 317-375.

[3] Г. А. Ломадзе, О представлении чисел суммами нечётного числа квадратов, Труды Тбилис. матем. ин-та 17 (1949), 281-314.

[4] -, О представлении чисел суммой $8 t+1$ квадратов, Труды Тбилис. гос. ун-та 179 (1976), 63-66.

[5] - On some entire modular forms of half integral weight for the group $\Gamma_{0}(4 N)$, in: New Trends in Probability and Statistics, Vol. 2 (Palanga, 1991), VSP, Utrecht, 1992, $57-67$.

[6] L. G. Mordell, On the representations of a number as a sum of an odd number of squares, Trans. Cambridge Philos. Soc. 22 (1919), 361-372.

МЕХАНИКО-МАТЕМАТИЧЕСКИЙ ФАКУЛЬТЕТ

ТБИЛИССКИЙ ГОСУДАРСТВЕННЫЙ УНИВЕРСИТЕТ

ПРОСП. И. ЧАВЧАВАДЗЕ 1

ТБИЛИСИ 380028

РЕСПУБЛИКА ГРУЗИЯ 